\title{
ÉVOLUTION DE LA FORMATION À DISTANCE DANS UNE UNIVERSITÉ BIMODALE
}

Serge Gérin-Lajoie, Claude Potvin

Lavoisier | « Distances et savoirs »

2011/3 Vol. 9 | pages 349 à 374

ISSN 1765-0887

ISBN 9782746237957

Article disponible en ligne à l'adresse :

https://www.cairn.info/revue-distances-et-savoirs-2011-3-page-349.htm

\section{Pour citer cet article :}

Serge Gérin-Lajoie, Claude Potvin« Évolution de la formation à distance dans une université bimodale », Distances et savoirs 2011/3 (Vol. 9), p. 349-374.

Distribution électronique Cairn.info pour Lavoisier.

(c) Lavoisier. Tous droits réservés pour tous pays.

La reproduction ou représentation de cet article, notamment par photocopie, n'est autorisée que dans les limites des conditions générales d'utilisation du site ou, le cas échéant, des conditions générales de la licence souscrite par votre établissement. Toute autre reproduction ou représentation, en tout ou partie, sous quelque forme et de quelque manière que ce soit, est interdite sauf accord préalable et écrit de l'éditeur, en dehors des cas prévus par la législation en vigueur en France. Il est précisé que son stockage dans une base de données est également interdit. 


\title{
Évolution de la formation à distance dans une université bimodale
}

\author{
Serge Gérin-Lajoie — Claude Potvin \\ Pavillon de l'Est \\ 2180, chemin Sainte-Foy \\ Local 2110 \\ Université Laval \\ Québec (Québec) G1V 0A6 \\ \{Serge.Gerin-Lajoie, Claude.Potvin\}@bsp.ulaval.ca
}

\begin{abstract}
RÉSUMÉ. Cet article présente une étude de cas décrivant l'évolution de l'Université Laval (UL), une université canadienne qui, d'un enseignement unimodal en présentiel, est progressivement devenue bimodale (en présentiel et à distance). Après en avoir dressé un court historique, les auteurs explorent l'évolution de la formation à distance (FAD) dans cet établissement sous divers aspects : le processus de développement des cours, les cinq principaux modèles de cours et le rôle de l'enseignant dans la phase de diffusion des cours. À partir du cadre conceptuel de Berge et Muilenburg (2001), les auteurs analysent les trois principaux freins au développement institutionnel de la FAD à l'UL. Les auteurs concluent par une évocation des perspectives d'évolution future de la FAD dans ce type d'établissement bimodal.

ABSTRACT. In this paper, a case-study, the authors are portraying the evolution of Distance Education (DE) at Université Laval, Canada, a formerly single-mode institution that eventually became dual-mode. After reporting the key historical events, four issues are explored: the course development process, the five main course models, and the role of faculty in course delivery. The last issue deals with Berge \& Muilenburg (2001) framework, in a discussion on how UL copes with the three main barriers to DE. As they conclude, the authors are wondering where the evolution of DE will bring dual-mode institutions.

MOTS-CLÉS: distance, bimodal, université, modèle, évolution, stade, TIC, classe, virtuel, collaboration, synchrone.

KEYWORDS: distance, dual-mode, university, model, evolution, stage, IT, class, virtual, collaboration, synchronous.
\end{abstract}

DOI:10.3166/DS.9.349-374 @ C Cned/Lavoisier 2011

D\&S - 9/2011. Où va la distance ?, pages 349 à 374 


\section{Introduction}

Pour de multiples raisons, à commencer par l'accessibilité, plusieurs établissements d'enseignement s'intéressent à la formation à distance depuis quelques années. Or, passer de l'unimodalité à la bimodalité n’est ni un processus simple, ni très bien documenté.

Cet article s'intéresse au cas de l’Université Laval (UL), une université canadienne francophone qui, d'unimodale au début des années 1980, est devenue progressivement bimodale, c'est-à-dire un « établissement d'enseignement supérieur qui offre des activités de formation en présentiel sur campus ou hors campus, et des activités de formation à distance. » (UL, 2011a, p. 2). En 2011, l’UL offre près de 600 cours à distance et plus de 50 programmes, en plus de ses 355 programmes offerts en présentiel. Ces données font de l’UL une des principales universités bimodales au Canada en 2011.

Après avoir exposé la problématique du passage à la bimodalité pour un établissement d'enseignement, cet article trace un tableau de l'évolution de la FAD à l'UL sous divers aspects : le processus de développement des cours, les modèles de cours, sur lesquels nous insisterons davantage, et le rôle de l'enseignant dans la phase de diffusion des cours.

À partir des données présentées, nous discutons du développement institutionnel de la FAD à l’UL à partir du cadre théorique de Berge et Muilenburg (2001), qui identifie les principaux obstacles dans le processus d'évolution organisationnelle vers la FAD. Nous concluons par une évocation des perspectives d'évolution future de la FAD à l’UL et dans les établissements universitaires bimodaux en général.

\section{Problématique}

De plus en plus d'établissements d'enseignement misent dorénavant sur la formation à distance, et notamment sur la formation en ligne. En 1999, GuriRosenblit prédisait le mouvement :

It is, 1 think, not unreasonable to predict that very few, if any, new distance teaching universities will emerge in the near future in the developed countries. It is more likely that dual-mode or consortia-type universities, providing distance education, will expand considerably in the near future. (p. 256)

Cette prédiction s’est avérée juste. Aux États-Unis, dans un sondage effectué par le Sloan Consortium auprès de 4523 établissements postsecondaires, on rapporte que 65 \% des 2512 établissements répondants considèrent la formation en ligne 
comme critique pour leur avenir, et que $31 \%$ des étudiants s'inscrivent à au moins un cours en ligne au cours de leur formation (Allen et Seamen, 2011) ${ }^{1}$.

L'appropriation partielle ou substantielle de la FAD par une majorité d'établissements d'enseignement semble donc incontournable, du moins dans sa version en ligne. Pourtant, dans leur forme pure, les systèmes de formation en présentiel et de FAD sont assez dissemblables. Comment alors la FAD se déploie-telle dans un établissement d'enseignement ? Est-ce en s'inspirant des pratiques des établissements unimodaux FAD ? C'est ce que prétend Bertrand (2010), qui évoque l'ubiquité des technologies de l'information et des communications (TIC) dans les deux univers :

On reconnaît maintenant l'apport indispensable des TIC dans le rapport au savoir, de même que l'impossibilité pour la formation en présentiel de relever le défi de la qualité et de l'accessibilité sans intégrer les TIC à ses dispositifs de formation, en prenant appui sur l'expertise de la FAD, aussi bien sur le plan de la pédagogie que sur le plan de la gestion d'un système de formation à distance. (Bertrand, 2010, p. 44)

Dans cette optique, certains établissements d'enseignement en présentiel se basent sur des directives telles celles que suggère Guri-Rosenblit (1999) pour créer une université à distance ${ }^{2}$. Beaucoup d'autres, rébarbatifs à l'univers de la FAD traditionnelle, sont plutôt séduits par les nombreux guides des meilleures pratiques issus du monde de la formation en ligne, tels ceux de Barker (2002), de SloanC (sloanconsortium.org/effective) ou de Marshall (www.utdc.vuw.ac.nz/research/ emm).

Outre ces instruments, on retrouve peu de directives spécifiques aux établissements bimodaux, si ce n'est quelques exceptions tel l'article de Hope (2005). Enfin, il existe peu de cas documentés du processus évolutif d'établissements universitaires vers la bimodalité, surtout du côté francophone. Dans cet article, nous présentons le cas de l’Université Laval.

Par ailleurs, Berge et Muilenburg (2001) proposent un cadre théorique intéressant pour observer le processus d'évolution vers la bimodalité. Dans leur article, ils présentent dix facteurs constituant des obstacles au développement de la FAD dans les organisations. Selon nous, les trois principaux facteurs identifiés par ces auteurs, facteurs pour lesquels nous disposons de données et d'observations, nous permettent d'analyser le cas de l'UL lors de la discussion.

1. Pour le Sloan Consortium, la formation en ligne implique qu'au moins $80 \%$ des contenus sont diffusés en ligne.

2. Voir l'annexe 1 de son ouvrage, intitulée Basic Guidelines for Initiating and Establishing a New Distance Teaching University. 


\section{Contexte historique de la formation à distance à l’Université Laval}

Depuis sa fondation à Québec en 1663, l’UL a toujours eu des activités de formation qui s'inscrivent dans la tradition universitaire d'enseignement en présentiel. C'est dans le milieu des années 1980 qu'elle se lance dans la FAD, afin de donner à la population canadienne ne résidant pas dans la région de Québec la possibilité d'avoir accès à l'enseignement universitaire en français. Ainsi, depuis 1984, une série d'évènements ont marqué l'évolution de la FAD à l'UL (UL, 2011b) :

- 1984 - Premier cours télévisé à distance ;

- 1992 - Premier cours par correspondance ;

- 1996 - Premier programme majeur à distance. Le certificat en planification financière personnelle (PFP) attirera en quelques années plus de 4500 étudiants dans un contexte de formation continue ;

- 1997 - Premier cours en ligne ;

- 2000 - Premier programme en ligne ;

- 2001 - Implantation de la plateforme WebCT ;

- 2004 - Première expérimentation d'un outil de classe virtuelle synchrone (CVS);

- 2005 - Dépôt d'un rapport interne sur la situation de la FAD à l'UL. Un programme de soutien financier au développement de la FAD est mis en place et chaque faculté est invitée à rédiger son plan de développement ;

- 2005 - La FAD est énoncée comme une priorité institutionnelle dans le discours de la rentrée du recteur de l'UL ;

- 2006 - La FAD, qui relevait jusqu'à ce moment de la Direction générale de la formation continue, relève dorénavant du Bureau de la formation à distance (BFAD), une nouvelle entité créée pour coordonner les efforts de développement et de diffusion de la $\mathrm{FAD}$;

- 2008 - La FAD devient objectif institutionnel dans le plan stratégique « Horizon $2012 »$;

- 2008 - Déploiement d'un service de CVS à l'échelle institutionnelle ;

- 2011 - Déploiement d'un nouvel environnement numérique d’apprentissage (ENA) qui remplace WebCT ;

- 2011 - Adoption d'une politique institutionnelle de la formation à distance.

Au fil des ans, la FAD s'est déployée dans toutes les facultés et est aujourd'hui partie intégrante de l'UL. En 2011, après plus de 25 années de développement en FAD, l'UL propose plus de 50 programmes de formation entièrement offerts à distance dans plus de 80 disciplines différentes. Ces programmes sont offerts au premier cycle (baccalauréats, certificats et microprogrammes) ainsi qu'aux cycles supérieurs (maîtrises et diplômes d'études supérieures spécialisées (DESS)). Le 
nombre de cours disponibles avoisine les 600 et $97 \%$ de ces cours sont offerts en ligne. La figure 1 présente l'évolution des inscriptions-cours ${ }^{3}$ en FAD dans cet établissement. Ainsi, en 2010-2011, la FAD a totalisé 36047 inscriptions-cours, ce qui représente près de $12 \%$ de l'ensemble des inscriptions-cours de l'université et fait de l'UL l'une des principales universités bimodales canadiennes.

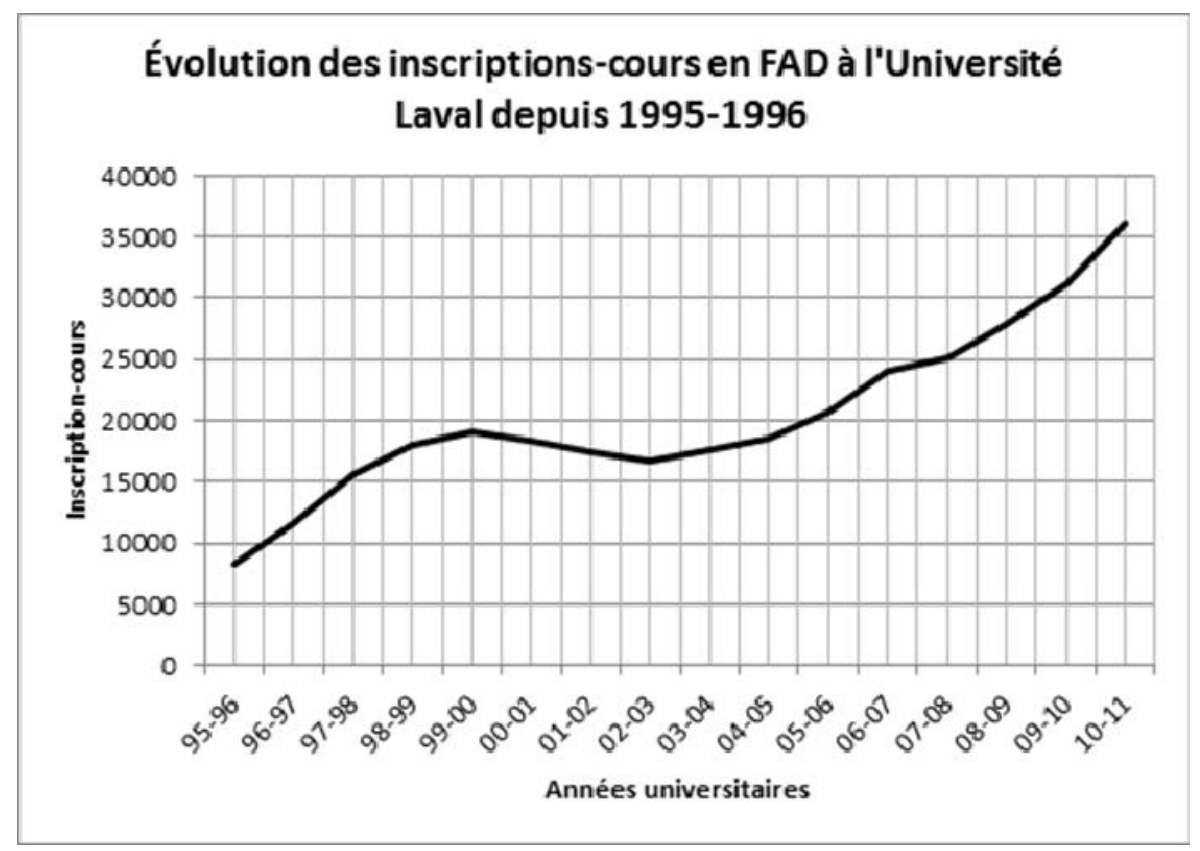

Figure 1. Évolution des inscriptions-cours en FAD à l'UL depuis 1995-1996

Au fil du temps, la définition de la FAD à l'UL a évolué. Celle qui est actuellement retenue est la suivante :

Système de formation qui permet à un étudiant d'apprendre seul ou en situation de collaboration, avec du matériel didactique approprié, avec différents moyens de communication et avec le soutien à distance de personnesressources. Cette formation se fait avec un minimum de contraintes d'horaire ou de déplacement, à l'exception des contraintes requises pour les évaluations sommatives des apprentissages. (UL, 2011a, p. 2)

3. Mesure de la fréquentation des cours à distance obtenue en cumulant le nombre d'inscriptions à chacun des cours. À distinguer des inscriptions à des programmes ou à des certificats, lesquelles concernent des « ensembles de cours ». 


\section{Le développement des cours}

Développer un cours à distance dans une université unimodale en présentiel tenait presque de l'hérésie en 1984, année où le premier cours à distance fut développé. Deux tendances se sont opposées pendant nombre d'années :

- reproduire en FAD les pratiques déjà éprouvées dans les cours en présentiel ;

- faire comme les établissements unimodaux en FAD.

La tradition d'enseignement en présentiel de l'UL influence beaucoup les pratiques pédagogiques dans le développement de cours à distance. Comme le soulignent Power et Vaughn (2010), citant les auteurs Goodyear (2001) et Jaffee (1998) :

The overall impression gleaned from numerous studies on faculty attitudes with regard to distance education and online learning is a strong desire to maintain intact a millennial tradition of on-campus teaching and learning. (p. 21)

Il est vrai que dans les premières années de l'apparition de la FAD à l'UL, cette tradition a fait sentir son poids : un enseignant ${ }^{4}$ disposait sensiblement des mêmes ressources humaines, financières et méthodologiques que pour développer un cours en présentiel.

Sous l'influence de certains conseillers ayant travaillé dans les établissements unimodaux en FAD, le processus de développement des cours à distance à l'UL a, au départ, beaucoup été calqué sur celui qui était utilisé dans ces établissements. Or, il existe de nombreuses différences fondamentales entre un établissement unimodal en FAD et un établissement en voie de se «bimodaliser », tel l'UL à cette époque. Le tableau 1 résume les principales différences.

Le processus de développement d'un cours à distance dans un établissement unimodal en FAD, fortement influencé par le caractère systématique de la technologie éducative, contraste avec le caractère plutôt artisanal et circonstanciel de la préparation de cours dans les universités unimodales nord-américaines offrant de la formation en présentiel. Pour l'essentiel, jusqu'aux années 2000, la tâche d'un enseignant de l'UL ayant pour mandat de développer un cours à distance a longtemps consisté à rédiger un document d'environ 500 pages, incluant des évaluations formatives et sommatives, le tout divisé en fonction d'une session de 15 semaines. Or, rédiger un tel document représente une tâche colossale, surtout s'il faut le faire seul à travers ses obligations régulières d'enseignement et de recherche.

Heureusement, pour l'assister dans cette tâche, l'enseignant a toujours pu compter sur l'assistance d'un conseiller pédagogique ainsi que d'une équipe technique. Toutefois, développer un cours en collaboration avec une équipe technopédagogique n’est pas nécessairement dans les habitudes des professeurs. En

4. Un enseignant représente toute personne à laquelle est confié un enseignement (professeur, chargé de cours, responsable de formation pratique, etc.) (UL, 2011c). 
effet, les enseignants qui ont la responsabilité de développer des cours à distance doivent agir non seulement à titre d'expert disciplinaire, leur force principale, mais aussi à titre de gestionnaire de projet.

\begin{tabular}{|c|c|c|}
\hline $\begin{array}{c}\text { Élément de } \\
\text { comparaison }\end{array}$ & $\begin{array}{c}\text { Établissement unimodal } \\
\text { FAD }\end{array}$ & Université Laval \\
\hline $\begin{array}{c}\text { Dates de début et } \\
\text { de fin de cours }\end{array}$ & $\begin{array}{c}\text { Variables (inscription } \\
\text { continue) }\end{array}$ & $\begin{array}{c}\text { Fixes } \\
\text { (les mêmes pour tous) }\end{array}$ \\
\hline $\begin{array}{c}\text { Processus de } \\
\text { développement }\end{array}$ & Systématique & Plus circonstanciel \\
\hline $\begin{array}{c}\text { Équipe de soutien } \\
\text { technopédagogique }\end{array}$ & $\begin{array}{c}\text { Lourde (i.e. complète avec } \\
\text { des spécialistes de tous les } \\
\text { domaines) }\end{array}$ & Lére \\
\hline $\begin{array}{c}\text { Libération } \\
\text { du professeur }\end{array}$ & $\begin{array}{c}\text { Pas nécessaire puisque c'est } \\
\text { sa tâche principale }\end{array}$ & $\begin{array}{c}\text { Parfois libération d'une } \\
\text { charge de cours pour } \\
\text { une session }\end{array}$ \\
\hline $\begin{array}{c}\text { Budget de } \\
\text { développement }\end{array}$ & Prévu dans le budget courant & $\begin{array}{c}\text { Petite subvention } \\
\text { institutionnelle }\end{array}$ \\
\hline
\end{tabular}

Tableau 1. Différences entre un établissement unimodal FAD et l'Université Laval quant au développement des cours FAD

Même si ce n'est qu'à compter de 2005 qu'un fonds de développement a officiellement été constitué, les facultés intéressées à développer des cours à distance ont pu, dès les années 1990, compter sur quelques subventions discrétionnaires, qui ont permis aux enseignants d'engager des assistants pour les aider à concevoir et rédiger le matériel didactique.

Un autre facteur facilitant pour le développement des cours à distance est l'arrivée des TIC à l'université, et en particulier l'adoption rapide par les enseignants en 2000 d'une plateforme de gestion des apprentissages, en l'occurrence WebCT. L'arrivée des TIC coïncide avec la constitution, vers la fin des années 1990, d'équipes de soutien technopédagogiques dans plusieurs facultés de l'UL, autrefois desservies par un service central. Au sein de ces équipes de soutien, les membres du personnel professionnel et technique possèdent des expertises variées dans plusieurs domaines : pédagogie universitaire, FAD, évaluation, production multimédia, technopédagogie, production vidéo, informatique, graphisme, etc. Actuellement, on peut faire le constat que tous les enseignants de l'UL ont accès à des spécialistes pour les conseiller et les assister dans leurs cours en présentiel ou à distance.

Au fil des années, s'appuyant sur les succès obtenus par quelques pionniers provenant de diverses facultés, les enseignants et les équipes de soutien technopédagogiques se sont appropriés le processus de développement d'un cours à 
distance, relativement rigide au départ, et l'ont adapté à leur contexte. Si bien qu'en 2008, le Bureau des services pédagogiques, une unité centrale, a proposé un processus révisé de développement et de diffusion d'un cours à distance, en collaboration avec le BFAD. Accompagnée de schémas et de guides, la rédaction de ces documents a permis de contextualiser le processus générique de développement et de diffusion d'un cours au caractère particulier de l'UL, tout en permettant une certaine souplesse.

Le processus révisé, disponible aux enseignants sur l'intranet du BSP, a aussi été présenté à la Table des praticiens de la $\mathrm{FAD}$, une communauté de pratiques du personnel professionnel de l'UL impliqué dans le développement et la diffusion de la FAD. Cette communauté, créée en 2009, permet des échanges entre les experts technopédagogiques qui soutiennent les enseignants et la mise en place de bonnes pratiques en FAD.

Ces dernières années, de nouvelles technologies ont été intégrées au processus de développement et de diffusion de cours, notamment les logiciels de production rapide, facilitant la préparation de capsules de formation multimédia, la classe virtuelle synchrone (CVS), les outils de collaboration tels le forum, le blogue et le wiki. De plus, depuis l'automne 2010, on assiste au déploiement d'un environnement numérique d'apprentissage (ENA) ${ }^{5}$ qui à terme remplacera WebCT. Cet environnement numérique permet le développement convivial et intégré de sites web de cours tant en présentiel qu'à distance. L'ensemble de ces technologies a pour point commun d'atténuer les frontières entre les activités d'enseignement en salle et celles qui doivent être développées à distance.

\section{Les modèles de cours à distance de l’Université Laval}

Au cours des vingt premières années de son histoire en FAD, l’UL a développé des pratiques d'enseignement et de formation de façon plus ou moins spontanée. Au fil du temps, des modèles ${ }^{6}$ de cours ont été esquissés. Un rapport interne produit en 2005 (UL, 2005) définissait cinq modèles de cours observés à l’UL :

- le modèle d'autoformation avec matériel imprimé (« Imprimé ») ;

- le modèle télévisé («TV »);

- le modèle d'autoformation avec matériel électronique («Électronique ») ;

- le modèle de formation en ligne avec apprentissage collaboratif («Collaboratif »);

- le modèle Téléformation, dit aussi Classe virtuelle synchrone (« CVS »).

5. Pour en connaître davantage sur ce nouvel environnement numérique d'apprentissage, voir : http://www.ena.ulaval.ca

6. Selon Depover (2009), un modèle est «la représentation plus ou moins formalisée d'un phénomène ou d'une situation » (p. 82). 
Dans la figure 2, les modèles sont situés selon deux axes qui mettent en évidence les différences quant à leurs caractéristiques temporelle et collaborative.

Dans les sections qui suivent, chaque modèle est présenté ainsi que son évolution dans le temps. Pour ce faire, différentes sources ont été utilisées: une étude exploratoire faite en 2008 (Gérin-Lajoie et Potvin, 2008), un sondage effectué auprès de la clientèle de l'UL en 2010 et diverses données institutionnelles (UL, 2011d).

L’étude exploratoire de 2008 avait pour but d'établir un portrait des pratiques en FAD à l'UL, notamment en vérifiant la présence, dans l'offre de cours, des cinq modèles de cours définis en 2005. Elle constitue à ce jour la seule source de données sur la présence de ces modèles à l’UL.

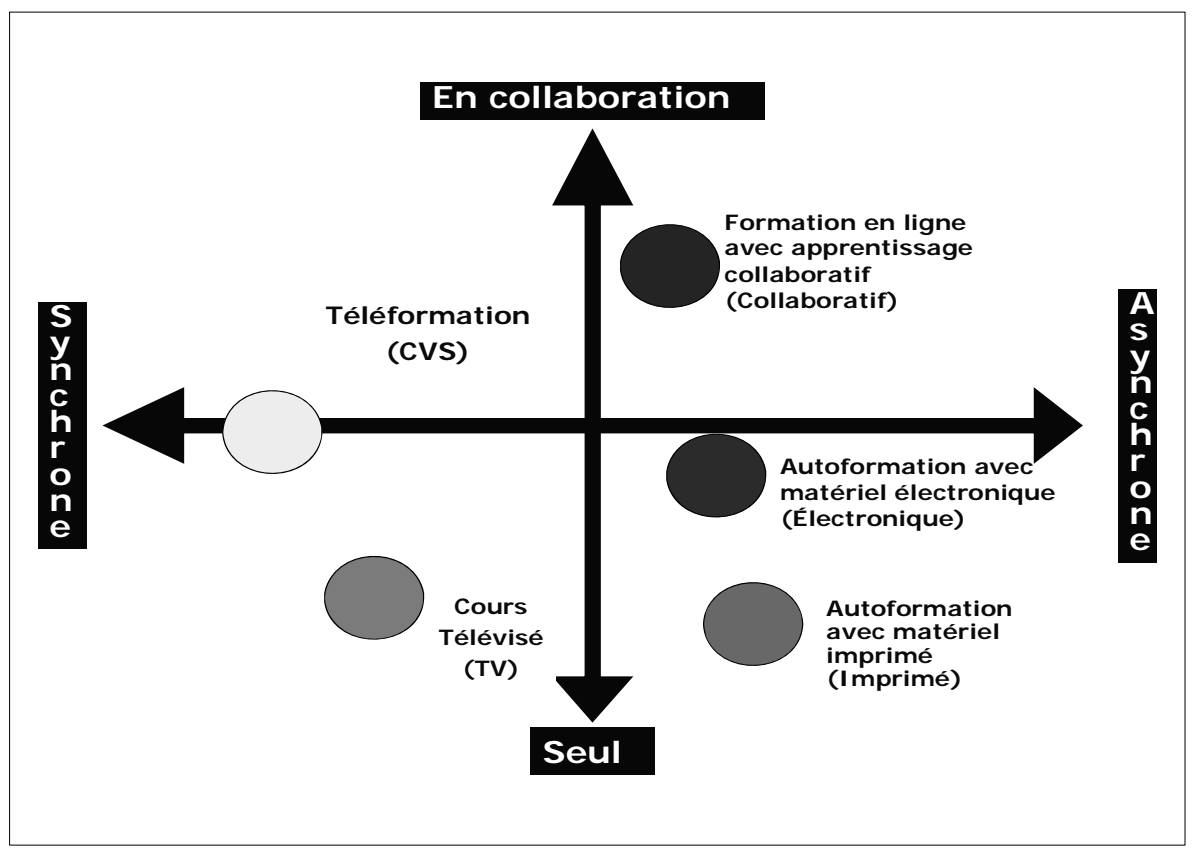

Figure 2. Les cinq modèles de cours FAD selon les dimensions de synchronicité et de collaboration

Dans le cadre de cette étude, un questionnaire a été envoyé à tous les enseignants responsables d'au moins un cours à distance. Cela a permis de recueillir des données sur les stratégies pédagogiques, les activités et outils de collaboration, le type de matériel didactique obligatoire, les supports et formats électroniques utilisés, les outils d'encadrement et le titre de la personne assurant l'encadrement des étudiants. 
Par des recoupements avec des données de nature administrative issues des bases de données internes, il nous a été possible de vérifier que l'ensemble des répondants constituait un échantillon significatif des différents modèles observés à l’UL. La figure 3 présente la proportion de cours par modèle, obtenue lors de l'étude.

Quant au sondage en ligne réalisé auprès de la clientèle étudiante de l’UL en 2010, il a été réalisé afin de cerner le profil type de ses étudiants à distance et vérifier si les services qui leur sont offerts correspondent à leurs besoins. 4586 étudiants provenant des 16 facultés et des études libres ont complété les 75 questions du sondage.

\section{Le modèle « Imprimé "}

Dans le modèle d'autoformation « Imprimé », les étudiants suivent un cours avec du matériel imprimé structuré et utilisent le téléphone pour rejoindre la personne qui les encadre. Ce genre de cours est essentiellement orienté vers des activités d’apprentissage individuelles et réalisées de façon asynchrone.

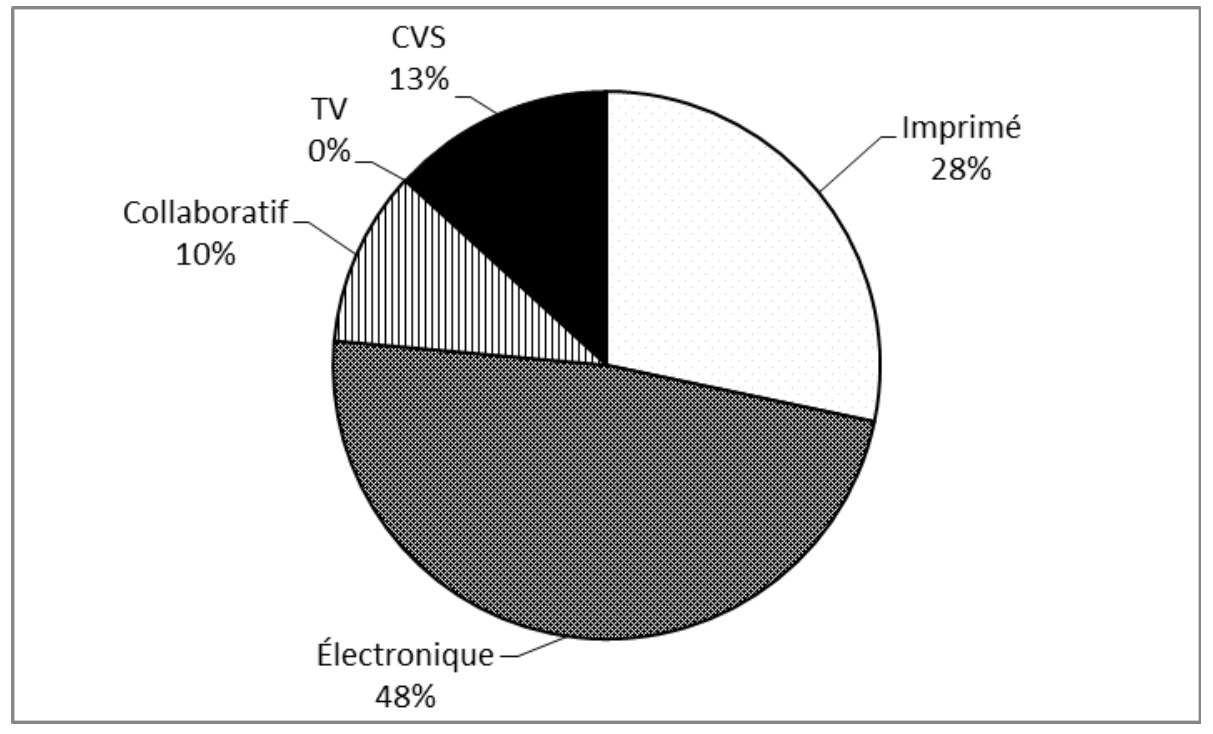

Figure 3. Proportion de chaque modèle de cours FAD à l'Université Laval en 2008

En 2008, nous avons constaté que ce modèle représente 28 \% de l’offre de cours à distance de l'UL. Aucun cours de ce modèle n'est offert aux cycles d'études supérieures, et il est celui qui offre le moins de diversité dans les stratégies pédagogiques. Les stratégies les plus courantes dans ce modèle sont la lecture de 
textes, la réalisation d'exercices et la résolution de cas ou de problèmes. Bien que le matériel didactique soit imprimé, on a constaté que $33 \%$ des cours de ce modèle utilisent un site web et des documents de type PDF (47\%) et DOC (23\%). Dans le même sens, on a remarqué, sans surprise que le courriel est utilisé dans 60 \% des cours de ce modèle, mais que les forums de discussion sont peu utilisés.

En 2011, on constate que ce modèle ne correspond plus qu'à $3 \%$ de l'offre de cours à distance de l'UL, comparativement à près de $100 \%$ en 1996 . En fait, les facultés qui utilisent ce modèle de cours ont progressivement mis en ligne leur matériel de cours.

La disparition prévisible du modèle «Imprimé » ne signifie pas pour autant que les étudiants sont prêts à abandonner le matériel didactique physique au profit du matériel entièrement numérique. Lors du sondage de 2010, une question portait sur le nombre de pages à partir duquel les répondants préféraient acheter du matériel physique plutôt que de lire à l'écran ou imprimer à la maison. À partir de 300 pages à lire, $74 \%$ des répondants préfèrent payer pour l'obtention du matériel imprimé. Dans les commentaires obtenus, 44 répondants précisaient que la lecture à l'écran peut être fastidieuse et que l'impression des documents à la maison peut s'avérer coûteuse en argent et en temps.

\section{Le modèle « $T V$ »}

Dans le modèle des cours télévisés («TV »), le matériel didactique est centré autour d'une série d'émissions télévisées de 30 à 60 minutes. La diffusion des émissions est assujettie à une grille horaire de diffusion. Les étudiants doivent donc regarder les émissions au moment de leur diffusion ou procéder à des enregistrements pour une écoute ultérieure. Les activités d'apprentissage proposées sont essentiellement individuelles. Dans ce modèle de cours, l'encadrement se fait essentiellement par téléphone, mais depuis environ 2002, le courriel et le forum sont aussi utilisés.

En 2008, nous nous sommes aussi demandé si ce modèle se destinait vers une fin annoncée. Nous savons que le nombre de crédits-étudiants à ce type de cours est en lente décroissance depuis 1998, passant de 6707 à 642 entre 1998-99 et 2008-09. Les cours qui sont encore diffusés vieillissent et sont tranquillement retirés de l'offre de cours. Selon nous, la décroissance de ce modèle s'explique essentiellement par deux raisons :

- la fermeture des services internes de production audio-vidéo à la fin des années 1990, forçant le recours à la production à l'externe, plus complexe à gérer ;

- la démocratisation de la production et de la diffusion sur Internet, autorisant plus de liberté dans les formats pour les enseignants et les équipes technopédagogiques. 
Par ailleurs, nous savons que les étudiants sont friands de matériel vidéo. Lors du sondage de 2010, plusieurs dizaines d'étudiants ont pris le temps de formuler un commentaire spécifique qui demandait d'accroître la quantité de matériel vidéo dans les cours en FAD, jugé plus intéressant que la lecture de textes et pouvant mieux répondre à certains types d'apprenants. Ils suggèrent plusieurs types d'utilisation de la vidéo: captation de professeurs ou de conférenciers, capsule d'informations, capsules de rétroaction, etc. Qui plus est, il est maintenant plus que jamais possible pour les étudiants de produire et de diffuser eux-mêmes des vidéos pour faire des exposés, récolter des éléments visuels sur le terrain ou faire des entrevues. Qu’ils soient produits par les enseignants ou les étudiants, on se rend compte que le matériel vidéo prend de plus de place dans les cours à distance de l'UL. Les statistiques internes le confirment: depuis 2005, la bande passante allouée à la diffusion vidéo à partir du serveur principal de cours - tous modes d'enseignement confondus - est passée de $6 \%$ en 2005 à $22 \%$ en 2010.

Selon nous, le développement de cours modèle « TV », avec plus de 10 heures de matériel vidéo par cours, se fera certainement plus rare. Cependant, nous constatons que le modèle « TV » a évolué vers un modèle de cours faisant toujours une grande place à la vidéo, mais plus ouvert dans son utilisation, sa production et sa diffusion. Dorénavant, on devrait parler de cours comportant des activités de télédiffusion ou webdiffusion.

\section{Le modèle « Électronique "}

Le modèle d'autoformation avec du matériel dit électronique ( Électronique ») est en quelque sorte une évolution médiatique des modèles précédents. Dans les cours de ce modèle, les étudiants accèdent à leur matériel didactique à partir d'un disque numérique ou d'un site web de cours. Ils se voient offrir des activités d'apprentissage un peu plus variées. Par exemple, il est possible d'aller visiter des sites web, de faire des exercices en ligne (quiz) ou des simulations et de participer à des échanges par courriel ou forum; ces outils étant aussi utilisés pour l'encadrement. Bien que la majorité des activités d'apprentissage dans ce genre de cours soient asynchrones, la collaboration est plus facile à organiser dans ce modèle.

Dans notre étude de 2008, le modèle "Électronique " représentait 48 \% de l'offre de cours à distance de l'UL, le pourcentage le plus important des cinq modèles. Il nous a semblé que l'utilisation des plateformes de gestion des apprentissages comme WebCT et leur effet intégrateur n'était pas étrangers à l'engouement des enseignants pour ce modèle.

A l'automne 2010, l'UL a commencé l'implantation d'une plateforme de cours de deuxième génération, intégrée dans un environnement numérique d'apprentissage (ENA). L'ENA permet l'accès aux services administratifs et pédagogiques à partir d'un seul endroit, et ce tant pour les cours en présentiel qu'à distance. Une autre des particularités de cette plateforme est d'utiliser une approche dite "par plan de 
cours ». Cette approche fait en sorte de structurer tous les sites web de cours en fonction de ce document essentiel. Outre une convivialité accrue par rapport aux anciennes plateformes, les enseignants peuvent utiliser un éventail de fonctionnalités pour répondre à leurs besoins pédagogiques. Au-delà du dépôt de n’importe quel type de documents électroniques, ils peuvent déployer des activités d'apprentissage et d'évaluation en utilisant des outils comme un gestionnaire d'équipes, l'évaluation par les pairs, la CVS, etc. Après deux trimestres de projet pilote, $92 \%$ des enseignants qui l'ont utilisé se sont dits satisfaits ou très satisfaits de leur nouvelle plateforme, comparativement à $80 \%$ du côté des étudiants (Larose, 2011), la principale critique étant que deux des quatre principaux navigateurs en vogue n'étaient pas encore supportés.

Un autre des facteurs pouvant expliquer la croissance du modèle «Électronique » est l'encouragement des enseignants à utiliser les TIC dans leur enseignement. En effet, depuis 1994, le Programme de financement pour l'élaboration de matériel pédagogique $(\mathrm{PADP})^{7}$ a aidé financièrement le développement d'applications pédagogiques des TIC (APTIC). À ce jour, ce sont près de $550000 \$$ qui ont été distribués à ce jour pour aider les enseignants à développer des tutoriels éducatifs, utiliser des animations graphiques, élaborer des présentations PowerPoint, développer des exerciseurs, des simulateurs, etc. Pour plusieurs enseignants, ces productions multimédias ont constitué un premier jalon technologique à partir duquel un cours à distance a pu prendre forme.

Par ailleurs, un autre facteur pouvant justifier la popularité du modèle «Électronique » est le niveau croissant de familiarité des étudiants avec les TIC. Lors du sondage de 2010, ces derniers ont été questionnés pour connaître leurs habiletés à utiliser les TIC. Résultat : 88 \% des répondants se considèrent de niveau soit expert ou intermédiaire, et $97 \%$ des répondants disposent d'une connexion Internet de niveau intermédiaire ou haute vitesse. Ces réponses démontrent qu'en 2011, les étudiants sont très à l'aise avec le modèle de cours « Électronique ».

\section{Le modèle "Collaboratif»"}

Le modèle de cours en ligne avec apprentissage collaboratif ( Collaboratif») diffère du modèle précédent par le fait que la majorité des activités d’apprentissage proposées aux étudiants se déroulent en mode de collaboration. Dans les faits, les concepteurs de cours dans ce modèle cherchent à créer des communautés d'apprentissage. Les outils de communication comme le courriel, le forum et le clavardage sont utilisés pour collaborer.

7. Les détails de ce programme sont présentés à : http://www.bsp.ulaval.ca/aide_finan ciere.php 
En 2008, nous avons constaté que ce modèle représentait $11 \%$ de l'offre de cours à distance de l’UL. De plus, le modèle « collaboratif » est celui qui a montré le plus de diversité dans les stratégies pédagogiques utilisées.

L'émergence de ce modèle à l'UL est notamment liée aux efforts de la Faculté des sciences de l'administration (FSA), pionnière dans l'utilisation d'Internet dans les cours à distance. Profitant du fait que les cours à distance de l'UL suivent le même calendrier trimestriel que les cours en présentiel, la FSA a développé plusieurs cours à distance où l'utilisation des TIC est obligatoire pour collaborer et réaliser des travaux d'équipe ; le but étant d'outiller les gestionnaires de demain à la réalité du monde des affaires du $21^{\mathrm{e}}$ siècle.

Dès 1996, avec la plateforme Virtual-U, et sur WebCT à partir de 2001, la FSA propose d'utiliser des outils de forum et de clavardage dans ses cours à distance. De plus, afin d'enrichir l'expérience des apprenants et après avoir exploré et expérimenté différentes technologies, cette faculté choisit de créer un premier bureau virtuel (environnement de collaboration privé spécifique pour les équipes de travail), comprenant des forums privés, un gestionnaire de fichiers, un calendrier et un espace de clavardage. En 2009, un nouveau bureau virtuel a vu le jour afin de répondre aux limites observées dans la première version et pour s'adapter aux nouveaux besoins des étudiants. Des fonctionnalités comme l'affichage de nouvelles, une bibliothèque de documents de travail avec du "versionning ", une banque d'hyperliens, un répertoire de contacts ainsi qu'un wiki ont été ajoutées. De plus, tout le processus de création et d'utilisation des bureaux virtuels est maintenant automatisé, minimisant ainsi les demandes de support des équipes techniques. Annuellement, on dénombre près de 1800 demandes de création de bureaux virtuels.

L'analyse des données de notre étude de 2008 nous permet de constater que ce modèle de cours dit collaboratif n'est plus confiné à la FSA. Des cours à distance du même genre se retrouvent désormais dans d'autres facultés de l'UL. Nous assistons à ce que Cleveland-Inness et Garrison (2010) identifient comme un virage vers la collaboration en FAD : "The post-industrial era of distance education is adopting many of the educational assumptions associated with interactive and collaborative learning” (p. 20).

L'aspect collaboratif de l'apprentissage n'est pas nouveau en éducation. Pour certains, il est même essentiel. Selon Loisier (2011) : «L'apprentissage collaboratif repose sur la théorie socioconstructiviste de l'apprentissage, selon laquelle l'apprenant apprend essentiellement par échange avec autrui ». Les possibilités qu'offrent les outils de communication facilitent l'élaboration d'activités d'échanges portant sur l'étude de cas, la résolution de problème, la discussion, le débat, etc. Quant à eux, les espaces de travail privés pour les équipes facilitent la gestion et la coordination des efforts de concertation, la rédaction, etc. Bref, ces outils et espaces de collaboration permettent de constituer ce que Wenger (1998) appelle des communautés de pratique ou d'apprentissage. 
Dans le sondage de 2010, 34 \% des répondants disent avoir réalisé des travaux d'équipes dans leurs cours à distance. De ces répondants, $80 \%$ reconnaissent que les travaux d'équipe leur permettent d'échanger des idées constructives, $65 \%$ reconnaissent que ces travaux en collaboration leur permettent de briser l'isolement lié à la distance, et $62 \%$ sont d'avis que les travaux avec leurs pairs rendent leurs cours à distance plus dynamiques. Par ailleurs, il faut garder à l'esprit que la réalisation de travaux en équipe à distance enlève de la flexibilité à la formule des études à distance. Dans le même sondage, $73 \%$ des répondants indiquent préférer travailler seuls et à leur rythme. Pour $45 \%$ des répondants, le travail en équipe vient ajouter des contraintes à leur horaire. Donc, nous en comprenons que l'intégration d'activités collaboratives dans les cours doit être dosée et justifiée.

Selon nous, l'émergence de ce modèle en FAD est intimement liée à une plus grande accessibilité aux TIC pour les enseignants, les étudiants et la société en général, facilitant ainsi de beaucoup les interactions synchrones et asynchrones. La mise en place de l'environnement numérique d'apprentissage à l'UL vient accentuer l'accessibilité à ces outils de communication. Au-delà des outils de communication intégrés dans l'ENA que sont le forum et la classe virtuelle synchrone, des fonctionnalités comme l'outil de gestion des équipes de travail, l'évaluation par les pairs et les espaces de travail privés, facilitent grandement la mise en place d'activités d'apprentissage collaboratives. Ainsi, dans une perspective où le travail en équipe est partie prenante de plusieurs habiletés ou compétences à développer dans les programmes de formation, il ne serait pas surprenant de constater une augmentation du nombre de cours de ce modèle dans les prochaines années.

\section{Le modèle " Téléformation » ou "CVS "}

Le dernier modèle défini en 2005 est le modèle "Téléformation », dit aussi « modèle avec classe virtuelle synchrone » ou «CVS ». Un outil de CVS permet de reproduire virtuellement une classe ; il comporte des fonctionnalités pour tenir des audioconférences, des visioconférences et pour partager le visuel d'écrans. Dans les cours de ce modèle, les étudiants sont invités à suivre une série de séances synchrones où différentes activités d'apprentissage sont proposées. Dans ce modèle de cours, l'encadrement se déroule en grande partie lors des séances synchrones, mais il est souvent complété par courriel ou forum.

Dans notre étude de 2008, ce modèle représentait $13 \%$ de l'offre de cours à distance de l'UL. Les cours du modèle "CVS » se retrouvaient en grande partie (86\%) dans les cours du premier cycle.

Aujourd'hui on constate que le modèle " CVS » s'est imposé graduellement à l'UL. Le tout a commencé en 2004 autour de l'Université virtuelle africaine avec laquelle l'UL avait établi un partenariat. En remplacement d'un système de vidéoconférence satellitaire jugé trop coûteux, un premier système de CVS 
(Interwise) fut testé avec succès, si bien qu’un certificat, puis un baccalauréat en informatique entièrement à distance furent éventuellement offerts à cette clientèle.

À compter de 2006, plusieurs facultés ont procédé à des expérimentations avec un outil de CVS. Puis, en 2007, un comité spécial recommandait de doter l'université d'un système de CVS (Elluminate), et affirmait à propos de la FAD que : « les systèmes synchrones permettent de varier les formules pédagogiques et d'aller au-delà des approches habituelles associées aux systèmes asynchrones " (p. 20). En effet, outre l'exposé magistral, toujours utilisé par les enseignants, le débat, la présentation de travaux en direct, le séminaire, la conversation et plus généralement les activités de nature interactive sont de plus en plus courantes dans plusieurs cours à distance de l’UL.

Entre 2008 et 2011, on note d'ailleurs une progression intéressante de l'utilisation de la CVS à l'UL. Elle est passée de 1500 rencontres en 2008-09 à 2400 en 2010-11, soit une augmentation moyenne de $21 \%$ par an. Quant au nombre de visionnements $^{8}$, il est passé de 38000 à 54000 pendant la même période, pour une augmentation d'environ $16 \%$. La fonction de visionnement des rencontres est donc très utilisée par les étudiants.

Ces chiffres ne font pas la distinction entre l'utilisation pour la FAD ou les autres utilisations telles qu'un invité dans un cours en salle, une réunion de gestion ou un simple test, mais on estime qu'à l'automne 2010, la CVS était utilisée par la FAD pour au moins $71 \%$ des rencontres. Cette même session, presque toutes les facultés ont utilisé la CVS au moins une fois. À l'automne 2010, c'est près d'un cours à distance sur 5 (19\%) qui utilisait au moins une fois la CVS pendant la session. Incidemment, c'est au $2^{\mathrm{e}}$ cycle que la CVS est la plus utilisée, soit dans $33 \%$ des cours, comparativement à $17 \%$ pour le premier cycle.

Pour quelques enseignants, la CVS est utilisée comme complément aux cours à distance qu'ils donnent déjà. Pour d'autres, la CVS est un bon moyen de débuter en FAD. Comme l'indiquent Anderson et al. (2003) et Keegan et al. (2005) : "This approach has the advantage of providing faculty with a sense of continuity in their teaching practice, as characterized by oral presentation and spontaneous interaction with students”. Dans le même sens, Power (2008) soutient que le temps nécessaire pour développer un cours à distance et son matériel didactique peut être réduit par l'utilisation d'un outil comme la CVS.

By adopting this technological solution, faculty and the researcher began to realize that the same low-level, front-end design as evidenced in on-campus courses could be effectively implemented, as opposed to administrationpromoted, high-level, front-end ID [Instructional Design] required by asynchronously-delivered online courses. Moreover, through the SDC [Synchronous Desktop Conferencing Environment] emulation of the familiar

8. Un visionnement consiste à revoir l'intégralité d'une rencontre, avec images et sons. À l’UL, toutes les rencontres sont enregistrées par défaut. 
classroom experience, faculty could compensate for the lack of front enddesigned material by substituting real-time classroom interaction and on-thespot feedback. (p. 508)

Du côté des étudiants, l'intérêt pour la CVS est présent, mais a des limites. Dans le sondage de 2010, seulement $37 \%$ des étudiants ont exprimé un désir d'avoir davantage de rencontres en CVS. Dans leurs commentaires, les étudiants ont mentionné son côté pratique pour la préparation aux évaluations, pour poser des questions, ou faciliter l'encadrement.

Nous sommes d'avis que le modèle CVS semble maintenant à l'UL pour rester, et probablement s'étendre à d'autres sphères que l'enseignement synchrone. On pense entre autres aux rencontres d'équipe à la demande, ou encore à l'encadrement individuel.

\section{L'enseignant et la diffusion}

L'encadrement est une composante fondamentale de la réussite d'une activité de FAD et ce, quel que soit le modèle utilisé. En 1999, un sondage auprès des étudiants en FAD ayant démontré des lacunes importantes dans l'encadrement des étudiants, une activité de perfectionnement pour les enseignants a été développée au cours de l'année suivante. Depuis, cette activité optionnelle a été offerte à en moyenne 30 personnes par an. En 2010, le sondage fait auprès des étudiants a démontré un bien meilleur taux de satisfaction quant à l'encadrement en général.

Il faut savoir qu'à l'UL, la personne assurant la fonction d'encadrement peut porter divers titres d'emploi. En 2008, notre étude sur le portrait de la FAD avait démontré que les professeurs, les chargés de cours ou d'enseignement et les auxiliaires d'enseignement (étudiants des cycles supérieurs) se partageaient à peu près de manière égale cette fonction (figure 4).

Bien que nous n'ayons pas de chiffres pour rendre compte de l'évolution de cette situation depuis 2008, plusieurs facteurs nous portent à croire que les professeurs sont de plus en plus actifs au moment de la diffusion du cours :

- l'augmentation des rencontres en CVS, le plus souvent animées par les professeurs responsables ;

- la contestation de l'appellation «tuteur » par le corps enseignant, au profit d'une appellation plus neutre (d'où l'expression «personne assumant l'encadrement ») ;

- un souci affirmé par certaines facultés les plus actives en FAD, d'offrir un service similaire tant à distance qu'en présentiel, où les professeurs doivent faire sentir leur présence de façon identique tant dans un cours en présentiel que dans un cours à distance ; 
- un processus administratif plus lourd depuis l'adoption en 2008 d'une convention collective du nouveau syndicat des auxiliaires administratifs, de recherche et d'enseignement.

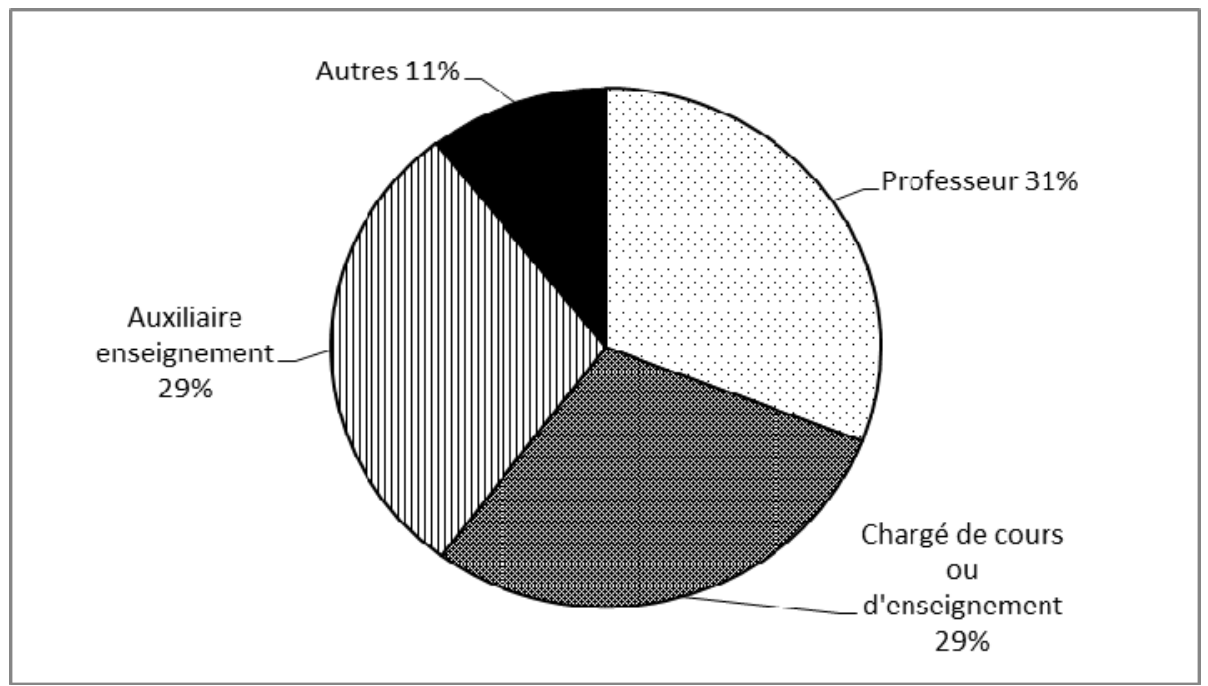

Figure 4. Distribution des titres d'emploi dans la fonction d'encadrement en 2008

Comme le mentionne Bertrand (2010) :

Alors qu'auparavant, le professeur [en FAD] n'avait que peu ou pas de contact direct avec les étudiants - ce travail étant l'apanage des tuteurs - cette division du travail change substantiellement, les conférences électroniques, par exemple, permettant aux étudiants de participer activement aux échanges avec les professeurs et les tuteurs. (p. 46)

Qui plus est, le processus de développement des cours se modifie depuis quelques années, au point où la phase de conception est parfois presqu'indissociable de la phase de diffusion, comme l'ont observé Henri et al. (2005). Dans certains domaines, l'accélération du renouvellement des connaissances, la proximité de l'information, la multiplicité des sources, l'instantanéité des accès amènent les professeurs à demeurer présents au moment de la diffusion de leurs cours. Ainsi, dans certains cours, il est de plus en plus courant que les enseignants décident d'inclure spontanément des éléments conjoncturels en complément aux éléments structurants, comme dans les cours en classe. La convivialité des nouveaux outils de production et de mise à jour de contenus, tels les outils de production rapide et le nouvel ENA, y est sans doute pour quelque chose. C'est ce que Bertrand (2010) confirme lorsqu'elle dit que : 
La production et la présentation du cours qui étaient deux processus distincts en FAD sont beaucoup plus intégrées dans la mesure où les contenus sont plus aisément modifiables lors de contacts avec les étudiants. (p. 47)

Quant aux technologies utilisées pour l'encadrement, notre étude de 2008 démontre que le courrier électronique est de loin l'outil le plus utilisé, soit dans $95 \%$ des cas. Même dans les cours du modèle «Imprimé », le courriel est utilisé dans $90 \%$ des cas. On remarque que le téléphone est encore utilisé dans $58 \%$ des cours tous modèles confondus, et dans $97 \%$ des cours du modèle "Imprimé ». Quant au forum, il est utilisé dans 52 \% des cours en général, mais environ deux fois moins dans les cours du modèle "CVS », ce qui n'est pas très surprenant étant donné l'interaction déjà possible dans les rencontres en synchrone. Enfin, si le clavardage (chat) est utilisé dans $10 \%$ des cours (incluant la CVS), le wiki et le blogue ne sont que rarement utilisés pour l'encadrement. Nous n'avons pas de raison de croire que les choses ont beaucoup changé depuis 2008 sur ce plan, si ce n'est que le téléphone standard est parfois remplacé par Skype. La figure 5 illustre la présence des outils d'encadrement par modèle.

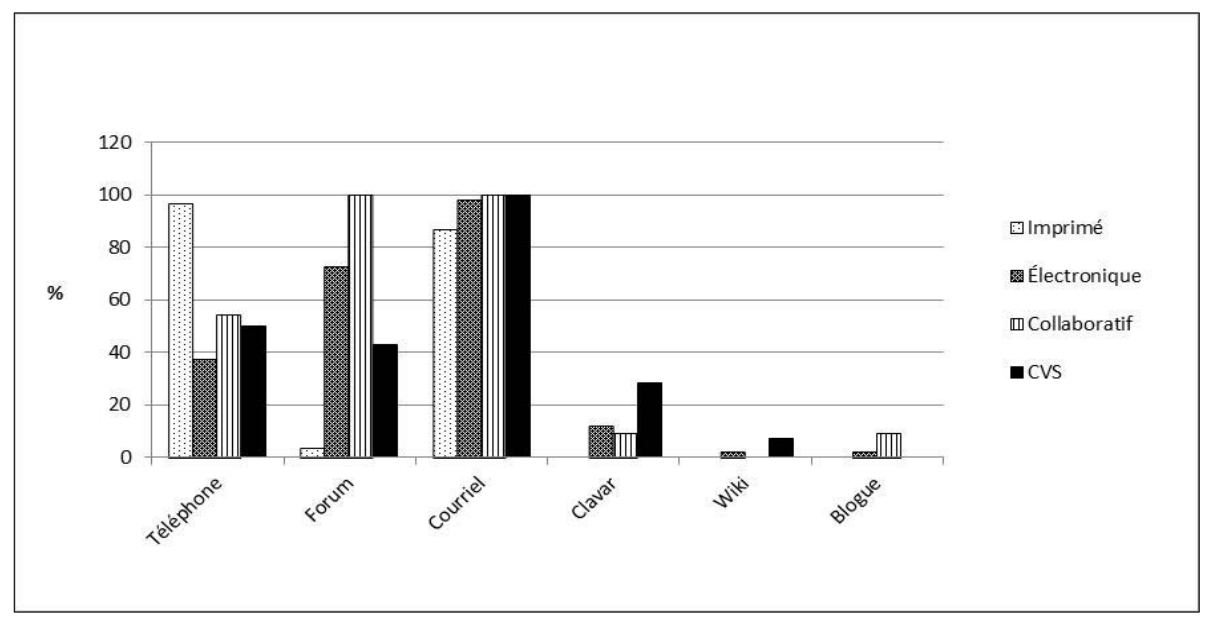

Figure 5. Outils d'encadrement par modèle en 2008

\section{Discussion}

Dans les lignes qui suivent, nous analyserons la présence, à l’UL, des trois principaux «facteurs-obstacles » au développement de la FAD identifiés par Berge et Muilenburg (2001), à la lumière des différentes observations et tendances explicitées précédemment. Ce sont ${ }^{9}$ :

9. Traduction libre. 
- le manque de reconnaissance à l'égard des enseignants ;

- la résistance organisationnelle au changement ;

- le manque d'expertise des enseignants et de l'équipe de soutien technique.

\section{Manque de reconnaissance à l'égard des enseignants}

Tel que souligné par Shea, Pickett et al. (2005), puis par Power et Vaughn (2010), le manque de reconnaissance à l'égard des enseignants qui s'impliquent en FAD, est un facteur constamment cité comme inhibant le développement de la FAD dans les établissements universitaires. Cette absence de reconnaissance se décline sous plusieurs dimensions: le temps nécessaire pour développer un cours, la considération du travail de préparation d'un cours dans leur tâche ou dans leur processus d'accréditation professionnelle, et l'absence de soutien financier pour le développement initial d'un cours ou pour sa mise à jour.

À l’UL, le processus de développement de cours est allégé et moins systématique que celui des établissements unimodaux en FAD. L'équipe technopédagogique qui accompagne un enseignant se charge de le conseiller et de l'aider à la demande. L'adoption rapide par les enseignants d'un outil comme la CVS dans les cours à distance tient probablement au fait qu'elle permet de répliquer les pratiques pédagogiques connues et donc de réduire le temps de développement. L'implantation d'un nouvel environnement numérique d'apprentissage simple et convivial va aussi dans cette direction, permettant à un enseignant d'élaborer plus rapidement son site web de cours.

Il est par ailleurs de plus en plus fréquent que les enseignants impliqués dans le développement d'un cours FAD soient libérés d'une charge d'enseignement pendant un trimestre pour travailler sur leur cours à distance. Cette pratique bouscule la culture d'enseignement universitaire puisque ce genre de libération est rarement accordée pour un cours en présentiel; mais pour certains enseignants, cette libération est une condition sine qua non pour qu'ils s'impliquent en FAD. Dans le même sens, nous observons également que certains professeurs incorporent le développement de leur cours à distance dans leur projet d'année de travaux et de recherches (sabbatique).

Le soutien financier au développement est un autre facteur à considérer. Depuis 2006, un programme de soutien triennal a été mis en place pour encourager financièrement le développement de la FAD à l'UL. Ce financement peut servir à engager des ressources d'enseignement supplémentaires, engager des auxiliaires qui assisteront un enseignant qui développe son cours à distance, financer la production de matériel didactique spécial (environnements d'apprentissage, tournage vidéos, etc.) ou procéder à l'acquisition d'équipement spécialisé nécessaire pour la mise à distance d'un cours. Un second programme, le PADP décrit précédemment, mis en place dès 1994 et accessible aux enseignants tant pour les cours en salle qu'à 
distance, est une autre source de financement qui permet d'encourager la production de matériel didactique innovateur.

Finalement, la reconnaissance du travail fait en FAD par les enseignants est en voie de s'accentuer. Depuis juin 2010, l'adoption de la Politique de Valorisation de l'enseignement et ses Dispositions relatives à l'évaluation de l'enseignement de l'UL (UL, 2011c) définissent un cadre pour une démarche concertée de promotion de la qualité et de l'amélioration en continu de l'enseignement offert dans les activités de formation de l'UL, ce qui inclut la FAD. Cette politique comprend plusieurs champs d'intervention, dont le programme des Concours des Prix d'excellence $^{10}$. Depuis 2003, ce programme reconnaît le travail des enseignants en $\mathrm{FAD}$ et ce, tant pour la production de matériel didactique exemplaire que pour l'excellence dans le déploiement de stratégies d'apprentissage, d'évaluation et d'encadrement.

\section{Résistance organisationnelle au changement}

Le deuxième plus important frein identifié par Berge et Muilenberg (2001) concerne le changement organisationnel. Tel que mentionné précédemment, la FAD n'est pas tombée des nues à l'UL. Dès la création des premiers cours, l'implication des professeurs, détenteurs des connaissances disciplinaires, a été nécessaire. Tout au long de la période d'accroissement du nombre de cours à distance dans les années 2000, les professeurs ont été partie prenante du processus de mise à distance de cours. Par ailleurs, plusieurs instances ont été créées, dont certaines de façon $a d$ hoc, afin de planifier et soutenir le développement de la FAD.

C'est le cas du comité pour la rédaction du rapport interne de 2005. L'identification de la FAD comme étant une priorité institutionnelle dans le discours de la rentrée du Recteur de l'UL en 2005 et la création du BFAD en 2006 ont constitué une première reconnaissance organisationnelle de l'importance de la FAD. Le BFAD a pour mandat général de voir à la bonne marche de la formation à distance à l'Université Laval. Plus spécifiquement, ce mandat concerne le développement de la $\mathrm{FAD}$ et sa promotion tant à l'interne qu'à l'externe; il concerne aussi tous les aspects de la diffusion de la FAD (information sur les cours et les programmes, gestion du site web de la FAD, qualité de l'encadrement pédagogique, technologique et administratif, etc.), l'organisation des examens en région ainsi que la gestion des partenariats en $\mathrm{FAD}$.

Toujours en 2006, on a vu la mise en place du premier plan triennal de soutien au développement de la FAD. Ce plan triennal, renouvelé en 2009, incite toutes les facultés de l'UL à élaborer un plan de développement en FAD. Ce sont ces plans de développement qui orientent les décisions en matière de la distribution des appuis

10. Pour en connaître davantage sur ce programme, voir: http://www.bsp.Ulaval.ca/ reconnaissance/prix_10_11/prix_10_11.php 
financiers. En 2008, l'inscription de la FAD en tant qu'objectif institutionnel dans le plan d'orientation de l'UL «Horizon 2012 » (UL, 2008) constituait un autre indicateur fort. En 2010, un comité consultatif sur la formation à distance a été mis sur pied, au sein duquel siègent des représentants de l'ensemble des facultés ; ce comité a comme mandat de conseiller le Vice-recteur aux études et aux activités internationales sur toute question relative au développement et à la bonne marche de la FAD à l'UL. Enfin, en 2011, le Conseil universitaire a adopté la Politique de la formation à distance (UL, 2011a) qui vient orienter les pratiques en FAD de l'UL. Résultant d'une consultation générale sur plusieurs années auprès de multiples instances composée de membres de la direction, d'enseignants et d'étudiants, cette politique vient officialiser un ensemble de définitions et de principes pour la plupart déjà en usage à l'UL ; elle ne devrait pas rencontrer de résistance lorsqu'elle sera mise application à compter de janvier 2012. L'ensemble de ces actions démontre que l'implantation de la FAD s'inscrit dans une dynamique de changement organisationnel à l’UL.

\section{Manque d'expertise technopédagogique}

Finalement, le troisième frein identifié par Berge et Muilenberg (2001) porte sur le manque d'expertise des enseignants pour faire de la FAD ainsi que le manque de soutien pédagogique et technologique pour les assister dans le développement et la diffusion de leur cours à distance. L'expertise nécessaire aux enseignants pour faire de la FAD s'analyse selon deux dimensions: celui de l'expertise spécifique à l'enseignement en FAD et celui de l'utilisation des TIC pour enseigner en FAD.

En ce qui concerne la première dimension, mentionnons que depuis le développement des tout premiers cours en FAD à l'UL, les enseignants ont toujours été soutenus de façon personnalisée par des conseillers spécialisés. Ces conseillers, près d'une vingtaine en 2011, se réunissent d'ailleurs autour de la Table des praticiens de la FAD pour partager les pratiques exemplaires. De plus, pour aider au développement de l'expertise des enseignants en FAD, l'UL a mis en place, depuis 2006, plusieurs activités de perfectionnement. Ainsi, selon son degré de familiarité avec la FAD, un enseignant peut assister à des activités d'initiation ou d'approfondissement. Finalement, tel que mentionné précédemment, une activité de perfectionnement spécifique a été mise en place pour former les enseignants qui sont appelés à encadrer des étudiants en situation de FAD.

C'est sans surprise que l'on constate que les premiers cours en FAD à l'UL se sont faits avec une intention de répliquer les pratiques qui avaient cours dans les établissements unimodaux en FAD. Les enseignants et les conseillers ne pouvaient que tenter de reproduire ce qui était connu en FAD. Il en résulte que les premiers cours développés étaient de types « Imprimé » et « TV » qui correspondent aux deux premières générations en éducation à distance de Moore et Kearsley (2005). 
Par ailleurs, tout comme dans les autres établissements universitaires québécois, les TIC se sont implantées dans les activités de formation durant les années 90. C’est pour encourager l'innovation dans l'utilisation de ces technologies que le PADP décrit précédemment a été créé. Lors de l'implantation de WebCT ou des autres systèmes de gestion des apprentissages, tous les enseignants ont pu s'approprier ces outils technologiques lors d'activités de perfectionnement. Certaines de ces activités abordent les aspects techniques des outils alors que d'autres traitent des bonnes utilisations pédagogiques autant dans les cours en présentiel que dans les cours à distance. Par exemple, lors de l'implantation du service de CVS, une activité d'initiation technique a été mise en place pour les novices et une autre pour ceux qui désirent approfondir les possibilités pédagogiques de la CVS. Ainsi, au fil de l'apparition de nouveaux outils technologiques, des activités de perfectionnement sont mises en place.

Depuis 2010, à l'occasion de l'implantation du nouvel environnement numérique d'apprentissage, un effort de formation important est déployé pour faire en sorte qu'un utilisateur novice soit autonome. Grâce à la simplicité de l'ergonomie et à la facilité à intégrer différents types de contenus. il en résulte que le temps de création d'un site web de cours est considérablement réduit.

Nous croyons que de la même manière que WebCT, ce nouvel environnement numérique d'apprentissage influencera grandement les pratiques pédagogiques en FAD à l'UL. Le premier effet a trait à la place centrale que prendre le «plan de cours » dans cet environnement. Ce document clé aura un effet structurant sur le design pédagogique des cours. Le second effet sera celui de la mise en place d'activités d'apprentissage et d'évaluation novatrices, par l'accès facilité aux outils et fonctionnalités. L'organisation du travail en collaboration ou l'évaluation du travail des pairs constituent de bons exemples. Évidemment, un enseignant à l'UL pourra utiliser cet environnement numérique tant pour ses cours en présentiel que ceux en FAD.

\section{Conclusion}

Dans cet article, nous avons parcouru l'évolution d'un établissement d'enseignement unimodal en présentiel vers un établissement d'enseignement bimodal en présentiel et à distance, en l'occurrence l'Université Laval. Cette évolution a été examinée sous divers aspects : le processus de développement des cours, les modèles de cours et le rôle de l'enseignant lors de la phase de diffusion des cours. Nous avons constaté :

- que le processus de développement des cours dans un établissement d'enseignement bimodal, s'il était relativement similaire en 1985 à celui d'un établissement d'enseignement unimodal en FAD, s'est adapté graduellement aux caractéristiques de ce genre d'établissement ; 
- que les modèles des cours développés se sont beaucoup diversifiés, que les TIC et internet y jouent dorénavant une place incontournable et que les modèles collaboratifs ainsi que la CVS prennent maintenant une place non négligeable ;

- que contrairement aux établissements d'enseignement unimodaux en présentiel, l'enseignant tend à demeurer actif dans la phase de la diffusion des cours dont il a la responsabilité.

Par ailleurs, en analysant le cas de cette université à la lumière des trois principaux facteurs-obstacles à la FAD identifiés par Berge et Muilenburg, nous avons constaté que pour chacun de ceux-ci, des initiatives ont été prises par l'UL pour les amoindrir et qu'un progrès considérable a été accompli.

La culture de l'enseignement en présentiel a certainement influencé la façon dont la FAD s'est développé à l'UL, notamment en cherchant à diminuer le temps de développement des cours, à optimiser les périodes de disponibilité de ses enseignants ou à les garder comme témoins actifs au moment de la diffusion. En revanche, l'implantation de la FAD a aussi fort probablement influencé les pratiques en présentiel ; que l'on pense à l'omniprésence des TIC en FAD, qui a contribué à l'apprivoisement des TIC, ou au caractère systématique du processus de développement d'un cours en FAD, qui constitue parfois pour un enseignant une formation pratique accélérée en pédagogie. Il semble donc que les distinctions entre les modes d'enseignement et de formation en présentiel et à distance s'amenuisent.

Bien qu'à ses débuts, la FAD à l'UL ait été appréhendée comme un mode d'enseignement marginal et de second ordre par certains membres du corps enseignant et certains gestionnaires, cette perception s'est estompée graduellement. Nous assistons de plus en plus à la convergence des pratiques des deux modes d'enseignement, même si le produit final comporte des caractéristiques distinctes. Comme le souligne Bertrand (2010) :

La FAD et la FEP [Formation en présentiel] sont ainsi appelées à se conjuguer pour susciter une université nouvelle fondée sur les valeurs traditionnelles, et dotée de moyens inusités et révolutionnaires. La mise en place de ce mode de formation universitaire original emprunte aux acquis de la FEP et de la FAD, par un processus d'hybridation, de contamination positive. Il les prolonge et les renouvelle, pour en faire une synthèse supérieure dans un modèle bimodal (p. 137).

L'adaptation d'un processus de développement de cours FAD qui colle mieux à la réalité d'un établissement bimodal y a été pour quelque chose. Mais selon nous, ce sont les initiatives structurantes telles la mise en place d'une aide financière au développement de la FAD, la mise sur pied d'instances fonctionnelles, consultatives ou décisionnelles spécifiques, l'adoption de politiques et règlements propres à la FAD et les accommodements faits pour faciliter la tâche d'un enseignant qui ont été les plus significatives pour l'acceptation de la FAD à l'UL. 
Pour imaginer ce que réserve l'avenir dans un établissement bimodal comme l’UL, plusieurs pistes sont à suivre. Sur le plan du développement des cours, jusqu’à quel point les nouveaux environnements numériques d’apprentissage viendront-ils accroître la convergence des pratiques d'enseignement et de formation en présentiel et à distance, notamment dans les cours des cycles supérieurs ? En ce qui concerne les modèles de cours, verra-t-on la formation hybride, mélangeant les deux modes dans un même cours, prendre de l'expansion ? Du côté du rôle des enseignants, comment leur présence se fera-t-elle sentir pendant la phase de diffusion ? Utiliseront-ils davantage la CVS, la téléphonie IP à la Skype, ou le web 2.0 ? Enfin, du point de vue institutionnel, quelle sera la proportion des activités FAD dans nos établissements d'enseignement bimodaux dans 10 ans ? Voilà autant de questions auxquelles nous serons attentifs dans les prochaines années.

\section{Bibliographie}

Allen I. E., Seamen J., Going the Distance, Online Learning in the United States, The Sloan Consortium, 2011. http://sloanconsortium.org/publications/survey/going_distance_2011

Barker K., FuturEd Inc., Canadian Recommended E-learning Guidelines (CanREGs), 2002. www.futured.com/pdf/CanREGs \%20Eng.pdf

Berge Z. L., Muilenburg L. Y., "Obstacles faced at various stages of capability regarding distance education in institutions of higher learning”, Tech Trend, vol. 46, n 4, 2001, p. $40-45$.

Bertrand L., Renouveler l'université, Les Presses de l’Université Laval, Québec, 2010.

Clevenland-Inness M. F., Garrison D. R., An introduction to Distance Education, New York, Routledge, 2010.

Depover C., La recherche en technologie éducative, Paris, Éditions des archives contemporaines, 2009.

Forgues J.-F., et al., Outils d'apprentissage et de communication synchrones, Rapport de travail, Québec, Université Laval, 2007.

Gérin-Lajoie S., Potvin C., « Modèles de cours à distance dans une université bimodale », Présentation au congrès du Réseau canadien pour l'innovation en éducation (RCIÉ/CNIE), Banf, AB, Canada, 2008.

Guri-Rosenblit S., Distance and Campus Universities - Tensions and Interactions, IAU New York, Press - Pergamon, 1999.

Henri F., Gagné P. et Maina M., «Étude d’usages : un choix méthodologique en vue de la conception d'une base de connaissances sur le téléapprentissage », S. Pierre (Ed.), Innovations et tendances en technologie de formation et d'apprentissage, Montréal, Presses internationales Polytechnique, 2005, p. 31-61.

Hope A., Factors for success in dual mode institutions. Vancouver: Commonwealth of Learning, 2005. http://www.col.org/SiteCollectionDocuments/06_DualModeInstitutions.pdf 
Larose Y., Un accueil très favorable, Au fil des événements, 2011. www.aufil.ulaval.ca/ articles/accueil-tres-favorable-32869.html

Loisier J., Les nouveaux outils d'apprentissage encouragent-ils réellement la performance et la réussite des étudiants en $F A D$ ?, Réseau d'enseignement francophone à distance du Canada (REFAD), 2011.

Marshall S., E-Learning Maturity Model, Victoria University of Wellington. www.utdc. vuw.ac.nz/research/emm/

Moore M.G., Kearsley G., Distance Education: a Systems View, Second edition, Belmont, Wadsworth Publishing Company, 2005.

Power M., Vaughn N., "Redesigning Online Learning for Graduate Seminar Delivery”, The Journal of Distance Education / Revue de l'Éducation à Distance, vol. 24, n 2, 2010.

Power M., “The Emergence of a Blended Online Learning Environment”, Journal of Online Learning and Teaching, vol. 4, $\mathrm{n}^{\circ}$ 4, 2008.

Shea P., Pickett A., et al., "Increasing access to Higher Education: A study of the diffusion of online teaching among 913 college faculty", The internationl review of research in open and distance learning, vol. 6, $\mathrm{n}^{\circ}$ 2, 2005.

Sloan-Consortium, Effective Practices. http://sloanconsortium.org/effective

Université Laval, Rapport du comité chargé de présenter à la vice-rectrice aux études un projet de politique institutionnelle sur le développement et le fonctionnement de la formation à distance, Rapport interne, 2005.

Université Laval, Horizon 2012: Orientations de développement de l’Université Laval, Québec, Université Laval, 2008. www2.ulaval.ca/fileadmin/cabinetrecteur/brochure_ horizon2012_3.pdf

Université Laval, Politique de la formation à distance, Québec, Université Laval, 2011a.

Université Laval, 25 ans de formation à distance à l'Université Laval, 2011b, www.bsp. ulaval.ca/fad_historique.php.

Université Laval, Politique de Valorisation de l'enseignement et Dispositions relatives à l'évaluation de l'enseignement de l'Université Laval, Québec, Université Laval, 2011c.

Université Laval, Sondage sur la formation à distance à l’Université Laval, Rapport analytique, Québec, 2011d.

Wenger E., Communities of practice: learning, meaning, and identity, New York, Cambridge University Press, 1998. 